Der Biologie-Preis 2010 wurde Frau Birte Höcker, Tübingen, für ihre herausragenden Beiträge zum Verständnis der Evolution von Enzymen verliehen.

\title{
Einblicke in die Mechanismen der Evolution durch Protein Design
}

\author{
BIRTE HÖCKER
}

Das heutige Leben basiert auf der chemischen Aktivität von Proteinen. Proteine gehören zu den Grundbausteinen der Zelle und nehmen an nahezu jedem zellulären Vorgang teil. Sie katalysieren chemische Reaktionen, erkennen und transportieren Stoffe, und geben der Zelle Struktur und Stabilität. Um diese komplexen molekularen Prozesse effizient und mit hoher Präzision auszuführen, nehmen Proteine in der Regel eine definierte dreidimensionale Struktur ein. Der Faltungsvorgang, der zu aktiven Strukturen führt, ist sehr komplex und leicht zu stören. Daher gibt es sowohl Faltungshelfer (Chaperone) in der Zelle, die den Vorgang unter-

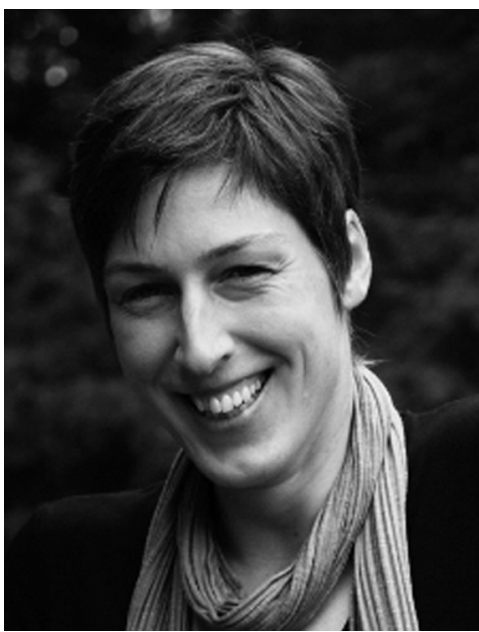

Birte Höcker, Gruppenleiterin am Max-Planck-Institut für Entwicklungsbiologie, Tübingen, Trägerin des Biologie-Preises 2010 stützen, als auch Mechanismen, die dazu führen, dass falsch gefaltete Proteine schnell abgebaut werden. Trotzdem beruhen viele Krankheiten auf der Mißfaltung von Proteinen, wie z. B. Alzheimer und Parkinson, was wiederum auf die Bedeutung des Faltungsprozesses für den Organismus hinweist. Zudem wissen wir heute, dass sich nicht jede beliebige Polypeptidkette faltet. Tatsächlich würde von einer Anzahl zufällig synthetisierter Proteinketten nur ein Bruchteil eine definierte Struktur einnehmen. Diese Problematik wird auch als das Faltungsproblem bezeichnet und ist eines der bedeutendsten ungelösten Probleme der Biologie.

Wie also konnte die Natur diese große Anzahl stabiler und funktionaler Proteine hervorbringen? Bei näherer Betrachtung von hochauflösenden 
Proteinstrukturen wird deutlich, dass das Problem in der Natur umgangen wird, indem unabhängig faltende Module (Domänen) immer wieder Verwendung finden. Diese strukturellen Grundeinheiten, die aufgrund ihrer räumlichen Anordnung in verschiedene Faltungstypen eingeteilt werden, bestehen meist aus etwa 100 aminosäurelangen Polypeptidketten. Bei Verwendung der üblichen 20 Aminosäuren ergibt sich eine Komplexität von $20^{100}$ Möglichkeiten, weitaus mehr als die Anzahl an Teilchen im Universum. Eine Domäne ist demnach ebenso zu komplex, um de novo entstanden zu sein. Wie also haben sich Domänen entwickelt?

Es wurde die Hypothese aufgestellt, dass die ersten gefalteten Proteine durch Fusion und Rekombination aus ursprünglichen Peptiden in einer RNA-basierten Welt entstanden seien. Diese Peptide nutzten zunächst Nukleinsäuren als Faltungsgerüste. Nach Fusion zu längeren Ketten stabilisierten sie sich gegenseitig und entwickelten sich über kleine, stabile Faltungseinheiten, über Domänen bis hin zu komplexeren Proteinen, die letztlich die Ribozyme als Katalysatoren ablösten ${ }^{1,2}$.

Ein sehr effizienter Weg, neue Proteine zu erzeugen, ist, bereits existierende stabile Faltungseinheiten mittels Genduplikation und Rekombination zu rekrutieren und anzupassen. Duplikation generiert dabei genetisches Material, welches ohne Verlust essentieller Gene durch Mutation und Selektion verändert werden kann. Rekombination, ein natürlicher Vorgang bei dem DNA-Stränge brechen und neu verknüpft werden, erhöht die Vielfältigkeit durch Neuordnungen der Gensequenzen. Durch diese Mechanismen kann die Komplexität von Proteinen schnell erhöht werden, während kleine Veränderungen und Anpassungen dagegen durch Punktmutationen erreicht werden. Letztlich entscheidet dann natürliche Selektion, ob diese zufälligen Veränderungen sich etablierten.

\section{Protein-Design nach Vorbild der Natur}

Die Natur hat unter Verwendung der oben beschriebenen Mechanismen eine Vielzahl Proteine hervorgebracht, die komplexe molekulare Prozesse effizient und präzise ausführen. Diese Mechanismen haben wir nun experimentell an Proteinen zweier weit verbreiteter Faltungen nachvollzogen, um zum einen die Hypothese der Evolution von Proteindomänen aus kleineren Einheiten zu prüfen und zum anderen ein verbessertes Verständnis der Abläufe hinsichtlich einer Anwendung im Protein-Design zu erhalten. Die Neukonstruktion oder das Design von Proteinen mit neuen, veränderten oder für eine Anwendung optimierten Eigenschaften wird angestrebt, seit- 
dem die gezielte Veränderung eines Protein-kodierenden Leserasters möglich ist. Das Studium der Evolution und ihrer Mechanismen kann daher als Grundlage für die Konstruktion neuer gefalteter und funktionaler Proteine dienen, da im Verlauf der Evolution Polypeptidketten kontinuierlich an spezifische, neue Funktionen angepasst werden ${ }^{3}$.

Für die Evolution der großen Faltungsfamilie der $(\beta \alpha)_{8}$ - oder TIMbarrel-Proteine werden zum Beispiel mehrere Genduplikationsereignisse diskutiert. In zwei $(\beta \alpha)_{8}$-barrel-Enzymen aus der Biosynthese der Aminosäure Histidin (HisA und HisF) wurde eine klare Sequenzsymmetrie gefunden, welche sich in einer eindeutigen strukturellen Ähnlichkeit der Proteinhälften widerspiegelt. Diese Beobachtung führte zu der Hypothese, dass diese beiden Enzyme durch zwei aufeinanderfolgende Duplikationsschritte aus einer Urhälfte hervorgegangen seien ${ }^{4,5}$. Diesen Evolutionsweg konnten wir im Labor rekonstruieren und aus zwei identischen Hälften des HisFEnzyms ein stabiles und gefaltetes Protein erzeugen (Abb. 1). Dazu wurden zwei Genkopien, welche für die C-terminale Hälfte von HisF kodieren, in tandem kloniert, das Protein heterolog in Escherichia coli exprimiert und charakterisiert ${ }^{6}$. Anschließend wurden stabilisierende Mutationen an der Kontaktfläche der Hälften eingefügt, so dass ein sehr stabiles, symmetrisches $(\beta \alpha)_{8}$-barrel entstand ${ }^{7}$.

Darüber hinaus konnten in einem weiteren Experiment Hälften von HisA und HisF neu kombiniert werden, die zusammen ein stabiles chimäres Protein bilden (Abb. 1). Dieses Ergebnis weist darauf hin, dass der Austausch von Halb-barrel-Domänen zur Diversifizierung der Proteinfamilie beigetragen haben kann. Die funktionellen Eigenschaften der Halb-barrel können dadurch neu kombiniert werden und mögen so zu neuen und komplexeren Funktionen geführt haben ${ }^{6}$. Wie schnell ein solches neues Proteingerüst eine Funktion akquirieren kann, konnte durch ein ,gerichtete Evolution"-Experiment gezeigt werden, indem mit wenigen Punktmutationen eine verwandte Funktion mit Wildtyp-ähnlicher Effizienz etabliert werden konnte ${ }^{8}$.

Ebenso kann auch die Rekombination von Fragmenten verschiedener Faltungen zu neuen Strukturen führen. Zum Beispiel finden sich große strukturelle Ähnlichkeiten zwischen dem oben beschriebenen Halb-barrel und Proteinen der Flavodoxin-ähnlichen Faltung ${ }^{9}$. Basierend auf dieser Beobachtung ersetzten wir den $\mathrm{N}$-terminalen Teil von HisF durch Teile des Flavodoxin-ähnlichen Proteins CheY. Durch Fusion der Fragmente $\beta 1$ und $\alpha 2-\beta 5$ aus CheY mit $\alpha 4-\alpha 8$ aus HisF entstand die sehr stabile Proteinchimäre $\mathrm{CheYHisF}^{10}$. Eine Kristallstrukturanalyse der Chimäre bestätigte die barrelähnliche Faltung und zeigte, dass die Struktur der Fragmente im 


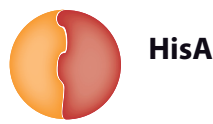

$\searrow$
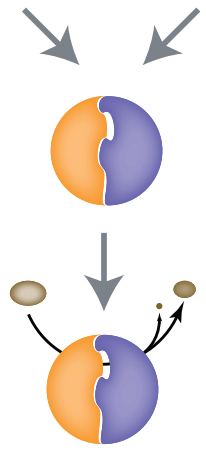

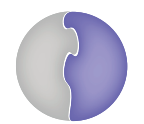

HisF
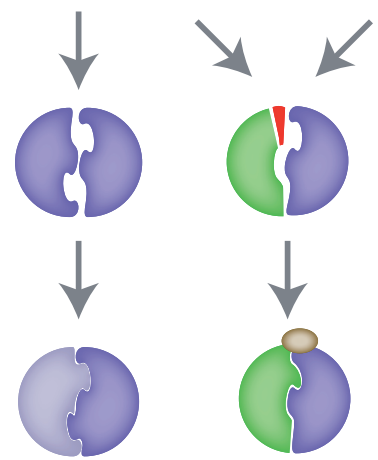

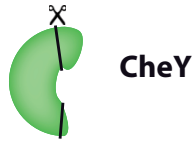

Rekombination
Optimierung/

Mutationen

Abbildung 1: Konstruktion von $(\beta \alpha)_{8}$-barrel-Proteinen durch Rekombination und anschließende Optimierung durch Punktmutationen. Links: Kombination von Halbbarrel-Fragmenten von HisA (orange) und HisF (blau), auf dessen Gerüst eine Aktivität etabliert wurde. Mitte: Duplikation und Fusion einer HisF-Hälfte mit anschließender Optimierung der Kontaktflächen. Rechts: Kombination von Fragmenten aus HisF (blau) und CheY (grün). Das neue Sekundärstrukturelement ist in rot dargestellt, mit anschließender Optimierung der Kontaktflächen und der Ligandenbindetasche.

neuen Kontext beibehalten wird. Jedoch deckte die Analyse auch einen unerwarteten neunten $\beta$-Strang auf, der vom C-terminalen Ende des Proteins gebildet wird und sich in das Innere des Barrel einfügt. Dieses neue strukturelle Element hebt Spannungen auf, die von einer suboptimalen Packung an der Kontaktfläche herrührt. Um die Kontaktflächen der Fragmente einander anzupassen und ein echtes achtsträngiges Barrel zu bauen, benutzten wir computergestütztes Design. Wenige gezielte Mutationen führten zu einem stabilen $(\beta \alpha)_{8}$-barrel, dessen vorhergesagte Struktur experimentell verifiziert werden konnte ${ }^{11}$. Weitere Optimierung dieser CheYHisF-Variante führte zudem zur Etablierung einer hochaffinen Ligandenbindungstasche (Abbildung 1).

\section{Schlußfolgerungen}

Diese Rekonstruktionsexperimente illustrieren einerseits, wie die Entstehung von Proteindomänen abgelaufen sein könnte, und zeigen andererseits den Einsatz derselben Mechanismen im Proteindesign. Wir können 
festhalten, dass die Konstruktion durch Duplikation und Rekombination zu neuen Proteingerüsten führt und dass wenige Mutationen zur Optimierung der Packung und Funktionalität ausreichen. Daraus lässt sich folgern, dass die Evolution von Proteinen aus kleineren Fragmenten in der Tat wahrscheinlich ist. Da unsere Experimente mit heutigen Proteinen durchgeführt wurden, können wir außerdem schließen, dass dieser dynamische Prozess auch heute noch stattfinden kann. Ingesamt zeigen die Ergebnisse, dass die Anwendung evolutionärer Mechanismen ein großes Potential für das Design von neuen Proteinen mit neuen Funktionen birgt.

\section{Literatur}

1. Lupas AN, Ponting CP \& Russell RB (2001) On the evolution of protein folds: are similar motifs in different protein folds the result of convergence, insertion, or relics of an ancient peptide world? J Struct Biol 134, 191-203.

2. Söding J \& Lupas AN (2003) More than the sum of their parts: on the evolution of proteins from peptides. Bioessays 25, 837-846.

3. Eisenbeis $S$ \& Höcker B (2010) Evolutionary mechanism as a template for protein engineering. J Pept Sci 16, 538-544.

4. Lang D, Thoma R, Henn-Sax M, Sterner R \& Wilmanns M (2000) Structural evidence for evolution of the $\beta / \alpha$-barrel scaffold by gene duplication and fusion. Science 289, 1546-1550.

5. Höcker B, Beismann-Driemeyer S, Hettwer S, Lustig A \& Sterner R (2001) Dissection of a $(\beta \alpha)_{8}$-barrel enzyme into two folded halves. Nat Struct Biol 8, 32-36.

6. Höcker B, Claren J \& Sterner R (2004) Mimicking enzyme evolution by generating new $(\beta \alpha)_{8}$-barrels from $(\beta \alpha)_{4}$-half-barrels. Proc Natl Acd Sci USA 101, 16448 16453.

7. Höcker B, Lochner A, Seitz T, Claren J \& Sterner R (2009) High-resolution crystal structure of an artificial $(\beta \alpha)_{8}$-barrel protein designed from identical half-barrels. Biochemistry 48, 1145-1147.

8. Claren J, Malisi C, Höcker B \& Sterner R (2009) Establishing wild-type levels of catalytic activity on natural and artificial $(\beta \alpha)_{8}$-barrel protein scaffolds. Proc Natl Acd Sci USA 106, 3704-3709.

9. Höcker B, Schmidt S \& Sterner R (2002) A common evolutionary origin of two elementary enzyme folds. FEBS Lett 510, 133-135.

10. Bharat TA, Eisenbeis S, Zeth K \& Höcker B (2008) A $\beta \alpha$-barrel built by the combination of fragments from different folds. Proc Natl Acd Sci USA 106, 3704-3709.

11. Eisenbeis S, Proffitt W, Coles M, Truffault V, Shanmugaratnam S, Meiler J \& Höcker $\mathrm{B}$ (2012) The potential of fragment recombination for the rational design of proteins. J Am Chem Soc 134, 4019-4022. 